\title{
Genome-wide identification and expression analysis of WRKY transcription factors in pearl millet (Pennisetum glaucum) under dehydration and salinity stress
}

\author{
Jeky Chanwala ${ }^{1 \dagger}$, Suresh Satpati ${ }^{1 \dagger}$, Anshuman Dixit ${ }^{1}$, Ajay Parida ${ }^{1}$, Mrunmay Kumar Giri ${ }^{2^{* \dagger}}$ and Nrisingha Dey ${ }^{1{ }^{*+}}$
}

\begin{abstract}
Background: Plants have developed various sophisticated mechanisms to cope up with climate extremes and different stress conditions, especially by involving specific transcription factors (TFs). The members of the WRKY TF family are well known for their role in plant development, phytohormone signaling and developing resistance against biotic or abiotic stresses. In this study, we performed a genome-wide screening to identify and analyze the WRKY TFs in pearl millet (Pennisetum glaucum; PgWRKY), which is one of the most widely grown cereal crops in the semi-arid regions.
\end{abstract}

Results: A total number of 97 putative PgWRKY proteins were identified and classified into three major Groups (I-III) based on the presence of WRKY DNA binding domain and zinc-finger motif structures. Members of Group II have been further subdivided into five subgroups (Ila-lle) based on the phylogenetic analysis. In-silico analysis of PgWRKYs revealed the presence of various cis-regulatory elements in their promoter region like ABRE, DRE, ERE, EIRE, Dof, AUXRR, G-box, etc., suggesting their probable involvement in growth, development and stress responses of pearl millet. Chromosomal mapping evidenced uneven distribution of identified 97 PgWRKY genes across all the seven chromosomes of pearl millet. Synteny analysis of PgWRKYs established their orthologous and paralogous relationship among the WRKY gene family of Arabidopsis thaliana, Oryza sativa and Setaria italica. Gene ontology (GO) annotation functionally categorized these PgWRKYs under cellular components, molecular functions and biological processes. Further, the differential expression pattern of PgWRKYS was noticed in different tissues (leaf, stem, root) and under both drought and salt stress conditions. The expression pattern of PgWRKY33, PgWRKY62 and PgWRKY65 indicates their probable involvement in both dehydration and salinity stress responses in pearl millet.

\footnotetext{
* Correspondence: mrunmay.giri@kiitbiotech.ac.in; nrisinghad@gmail.com; ndey@ils.res.in

Nrisingha Dey is the corresponding author and Mrunmay Giri is the co-corresponding author.

†Jeky Chanwala and Suresh Satpati contributed equally to this work.

${ }^{\dagger}$ Mrunmay Kumar Giri and Nrisingha Dey contributed equally to this work.

${ }^{2}$ School of Biotechnology, Campus 11, KIIT (Deemed to be) University, Patia,

Bhubaneswar, Odisha 751024, India

${ }^{1}$ Institute of Life Sciences, NALCO Nagar Road, NALCO Square,

Chandrasekharpur, Bhubaneswar, Odisha 751023, India
}

(c) The Author(s). 2020 Open Access This article is licensed under a Creative Commons Attribution 4.0 International License, which permits use, sharing, adaptation, distribution and reproduction in any medium or format, as long as you give appropriate credit to the original author(s) and the source, provide a link to the Creative Commons licence, and indicate if changes were made. The images or other third party material in this article are included in the article's Creative Commons licence, unless indicated otherwise in a credit line to the material. If material is not included in the article's Creative Commons licence and your intended use is not permitted by statutory regulation or exceeds the permitted use, you will need to obtain permission directly from the copyright holder. To view a copy of this licence, visit http://creativecommons.org/licenses/by/4.0/ The Creative Commons Public Domain Dedication waiver (http://creativecommons.org/publicdomain/zero/1.0/) applies to the data made available in this article, unless otherwise stated in a credit line to the data. 
(Continued from previous page)

Conclusion: Functional characterization of identified PgWRKYs can be useful in delineating their role behind the natural stress tolerance of pearl millet against harsh environmental conditions. Further, these PgWRKYs can be employed in genome editing for millet crop improvement.

Keywords: Pearl millet, WRKY transcription factors, Cis-regulatory elements, Synteny, Abiotic stress

\section{Background}

Global warming has a substantial impact on sustainability of the crop plants. Agricultural production is becoming more vulnerable due to climate variability [1]. Climate change associated environmental problems such as soil erosion, drought, flood, high temperature and an altered pattern of precipitation results in low and erratic crop yield [2]. Alongside, the increasing human population with intense urbanization affects the crop production and cultivated land area negatively. To ensure future food security, it is an utmost need for promoting the cultivation of major crops along with naturally adapted crops like millets, which can sustain under harsh environmental conditions [3].

Pearl millet (Pennisetum glaucum), syn. Cenchrus americanus, is one of the most widely grown crop in the arid and semi-arid tropical regions of Africa and South-east Asia including India. It serves as one of the staple food for millions of poor people and is also being used extensively for fodder and fuel [4]. It is highly resilient and well adapted to severe abiotic stresses including elevated temperature, drought and high soil $\mathrm{pH}$. A mean annual rainfall of around $250-300 \mathrm{~mm}$ is sufficient for pearl millet grain production, where most of the other important crops like rice, wheat, sorghum and maize are likely to fail [5]. Apart from this advantage of growing in adverse environmental conditions, pearl millet also has high nutritional index compared to rice, wheat, sorghum and maize. Pearl millet contains $8-19 \%$ protein, low starch, high fiber and essential micronutrients such as iron and zinc $[6,7]$. Due to these characteristics, worldwide attention is now focused on pearl millet cultivation to cope up with climate change and food insecurity [8].

Abiotic stresses cause damages to crop productivity and it accounts for more than 50\% agricultural production losses. Drought and salinity are two major constraints having a multidimensional impact on growth and productivity of the crops as they result in depleted groundwater tables, photosynthetic inhibition, reduced membrane protein stability and changes physiochemical properties of soil [9]. It has been seen that a $10 \%$ drop in rainfall results in an average of $4.2 \%$ decrease in cereals yield [10]. All water constraints, including drought results in $15-30 \%$ of agricultural yield losses [11]. Likewise, salinity also drastically affect crop productivity. On average, higher than normal salinity conditions prevail in $20 \%$ of cultivated and $33 \%$ of irrigated land globally [12].
All-important glycophytic crop plants reduce their average global yield by $50-80 \%$ under moderate salinity conditions $[13,14]$.

Plants have adapted several ways to escape such environmental stresses by employing several integrated transcriptional and hormonal factors. Specific transcription factors (TFs, the regulatory proteins) bind to the respective cognate cis-elements present in the promoter region of their target genes and modulate the expression level of genes under particular stress conditions. Such "cis-trans" interactions manifest significantly for controlling the plant survival under adverse environmental conditions [15]. In plants, several TF families have been reported namely ABRE-binding factor (ABF)/ABA-responsive-elementbinding (AREB) [16], ethylene responsive element binding factors (ERF) [16], DREB [17], NAC [18], AP2/ERF [19], WRKY [20], MYB [21], MYC [22] and basic domain leucine zipper (bZIP) [23] etc.

Structurally, WRKY transcription factors have conserved WRKY domain with signature sequence (WRKYGQK) along with zinc-finger motif (C-C, H-H/C) [24]. Broadly, WRKY transcription factors are classified into three major groups based on the number of WRKY domains and arrangement of the zinc-finger motif. Group I protein sequences contain two WRKY domains (at both $\mathrm{N}$ and $\mathrm{C}$ terminal) along with $\mathrm{C}_{2} \mathrm{H}_{2}$ zinc-finger motif $\left(\mathrm{CX}_{4-5} \mathrm{C}\right.$ $\mathrm{X}_{22-23} \mathrm{HXH}$ ). Group II proteins have only one WRKY domain followed by a $\mathrm{C}_{2} \mathrm{H}_{2}$ zinc-finger motif $\left(\mathrm{CX}_{4-5} \mathrm{CX}_{22-23} \mathrm{HXH}\right)$. Further, Group II proteins are classified into five subgroups, namely IIa, IIb, IIc, IId and IIe based on sequence characteristics and phylogenetic analysis. Like Group II proteins, Group III proteins also have a WRKY domain. However, instead of $\mathrm{C}_{2} \mathrm{H}_{2}$ motif, a $\mathrm{C}_{2} \mathrm{HC}$ zinc-finger motif $\left(\mathrm{CX}_{7} \mathrm{CX}_{23} \mathrm{HXC}\right)$ is conserved in Group III members [24-26]. Recent studies have assigned WRKY proteins possessing WRKY domain with no or partial zinc-finger motif structure to a separate group (Group IV; uncharacterized) [27-31].

Considering that WRKY TF is one of the key biological regulators, several studies have characterized their role in various plant species like foxtail millet, wheat, cotton and grapevine etc. [28-30, 32-36]. However, no such studies have been reported that may provide extensive insights about the role of WRKY TFs in pearl millet (P. glaucum). In this study, we have undertaken approaches for 
genome-wide identification of putative WRKY proteins present in pearl millet, their classification into different groups, chromosomal distribution, presence of conserved motifs, phylogenetic relationship, and sequence homology with WRKY family members of Arabidopsis thaliana, Oryza sativa (rice), and Setaria italica (foxtail millet). Further, we analyzed the relative expression profile of WRKY genes in different plant tissues and in response to drought and salinity stresses. The findings of this study will facilitate us to understand the mechanism behind the natural adaptation of pearl millet under abiotic stress. Also, candidate pearl millet WRKY genes can be employed in designing genetically improved millet for boosting agricultural production.

\section{Results}

Identification of the WRKY transcription factors in P. glaucum The HMMSCAN search resulted in the identification of 97 WRKY (PgWRKY1 to PgWRKY97) transcription factors from the complete proteome database of P. glaucum.
Further, protein sequence length, molecular weight (MW), isoelectric point (pI) and other indexes were analyzed for all identified 97 PgWRKYs of P. glaucum. We observed that the sequence length of the WRKY proteins varies from 123 amino acids (PgWRKY16) to 1394 amino acid residues (PgWRKY85). Their MW ranges from 13.732 to $156.285 \mathrm{kDa}$, and the $\mathrm{pI}$ ranges from 4.49 to 10.29 (Additional file 1).

\section{Classification of PgWRKY proteins and phylogenetic analysis}

The PgWRKY proteins were examined for conservation of the WRKY domain using multiple sequence alignment. As shown in Fig. 1, the sequences with amino acid conservation were shown in blue to red colour index where blue indicates the least and red means highly conserved patches. Multiple sequence alignment showed high conservation of "WRKYGQK" motif and "zinc-finger motif" in all identified PgWRKYs. Identified 97 PgWRKY proteins were classified into three groups

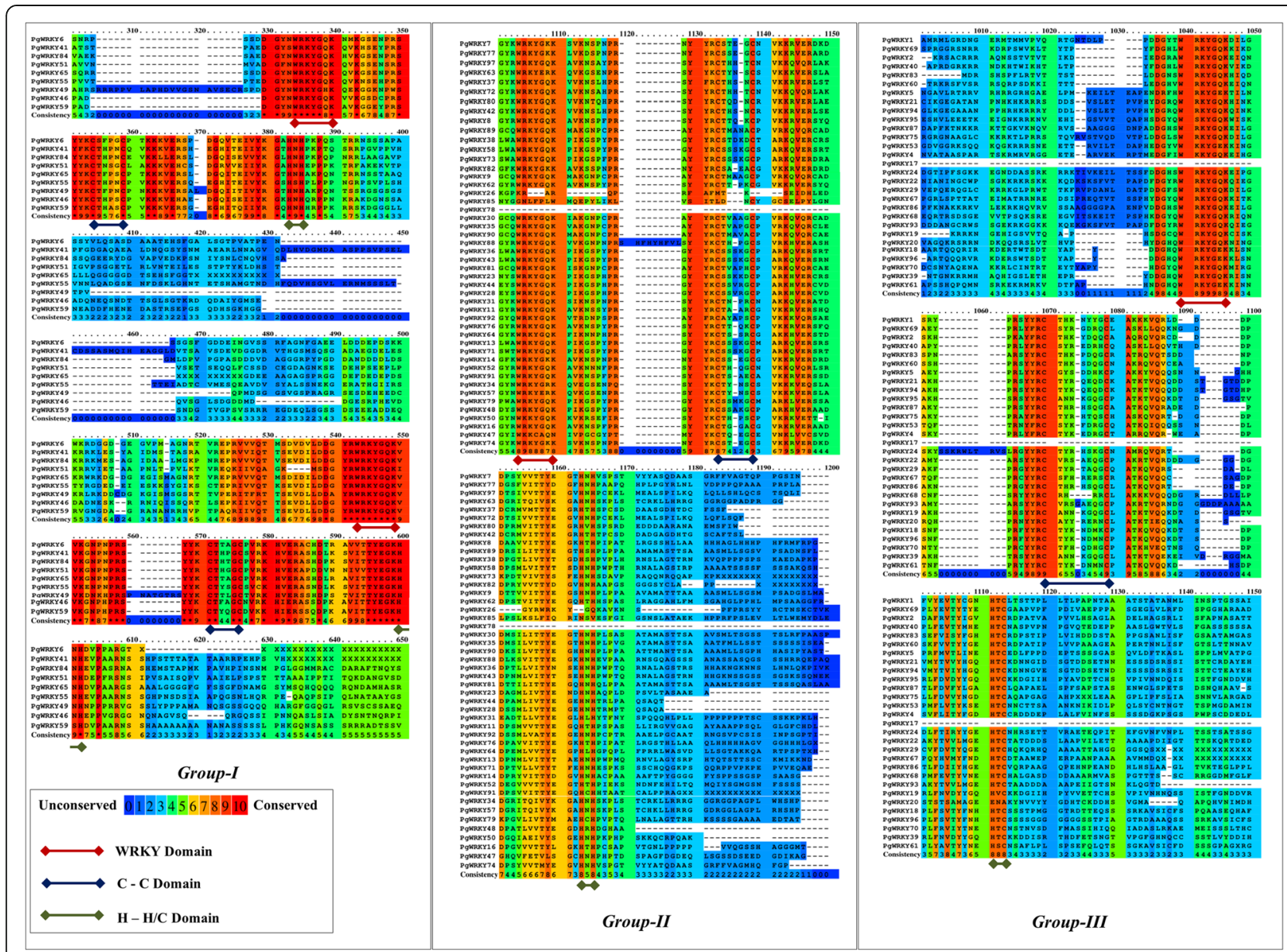

Fig. 1 Multiple sequence alignment of identified PgWRKY proteins. The amino acid conservation is shown in shaded colours, while domain conservation is shown through underline colours. The shaded colours indicate low to high residue conservation i.e., blue to red. The domain conservation for WRKY, C-C and H-H/C domains are shown through underline red, blue and green colour respectively 
based on the number of WRKY domains and structure of zinc-finger motif. Among the identified 97 PgWRKYs, we observed 9 PgWRKYs belongs to Group I; 47 PgWRKYs belong to Group II (forming the largest group); 29 PgWRKYs belong to Group III. Furthermore, we did not observe an intact zinc-finger motif in remaining 12 PgWRKYs. This is consistent with earlier studies conducted on Setaria italica, Gossypium hirsutum and Musa balbisiana [28, 29, 32]. Hence, these 12 PgWRKYs were kept in a separate group (Group IV; uncharacterized). Most of the PgWRKYs contain the conserved "WRKYGQK" motif, whereas few PgWRKYs have slight variations in their signature motif (Additional file 2).

A phylogenetic study was performed to analyze the evolutionary relationships among the WRKY families of A. thaliana, O. sativa, S. italica and P. glaucum. A total of 379 WRKY proteins including 72 from A. thaliana, 105 from O. sativa, 105 from S. italica, and 97 from $P$. glaucum were used to construct a phylogenetic tree as described in the method section. As shown in Fig. 2, all
379 WRKYs were clustered across major clades. We observed WRKY members belonging to a specific group (I, II, III) of all analyzed species were also clustering to the same clade (highlighted in Fig. 2).

\section{Chromosomal distribution and structure analysis of PgWRKY genes}

Identified PgWRKYs were mapped on seven chromosomes of $P$. glaucum (Fig. 3). Eighty-eight PgWRKYs were unevenly distributed across the $P$. glaucum genome. Remaining 9 PgWRKYs were not mapped due to unavailability of chromosomal coordinates in the genome database. Most of the PgWRKYs were abundant on 1st (22 genes; $\sim 23 \%$ ) and 6th (21 genes; $\sim 22 \%$ ) chromosomes whereas least were found on 5th and 7th (6 genes each; 6\%) chromosomes. A total number of $19 \mathrm{PgWRKYs}$ were located at the telomere region of chromosome 1, while 17 $P g W R K Y s$ were traced at the centromere region of chromosome 6. WRKY members of all groups were present on all chromosomes except chromosome 2 and 3 ,

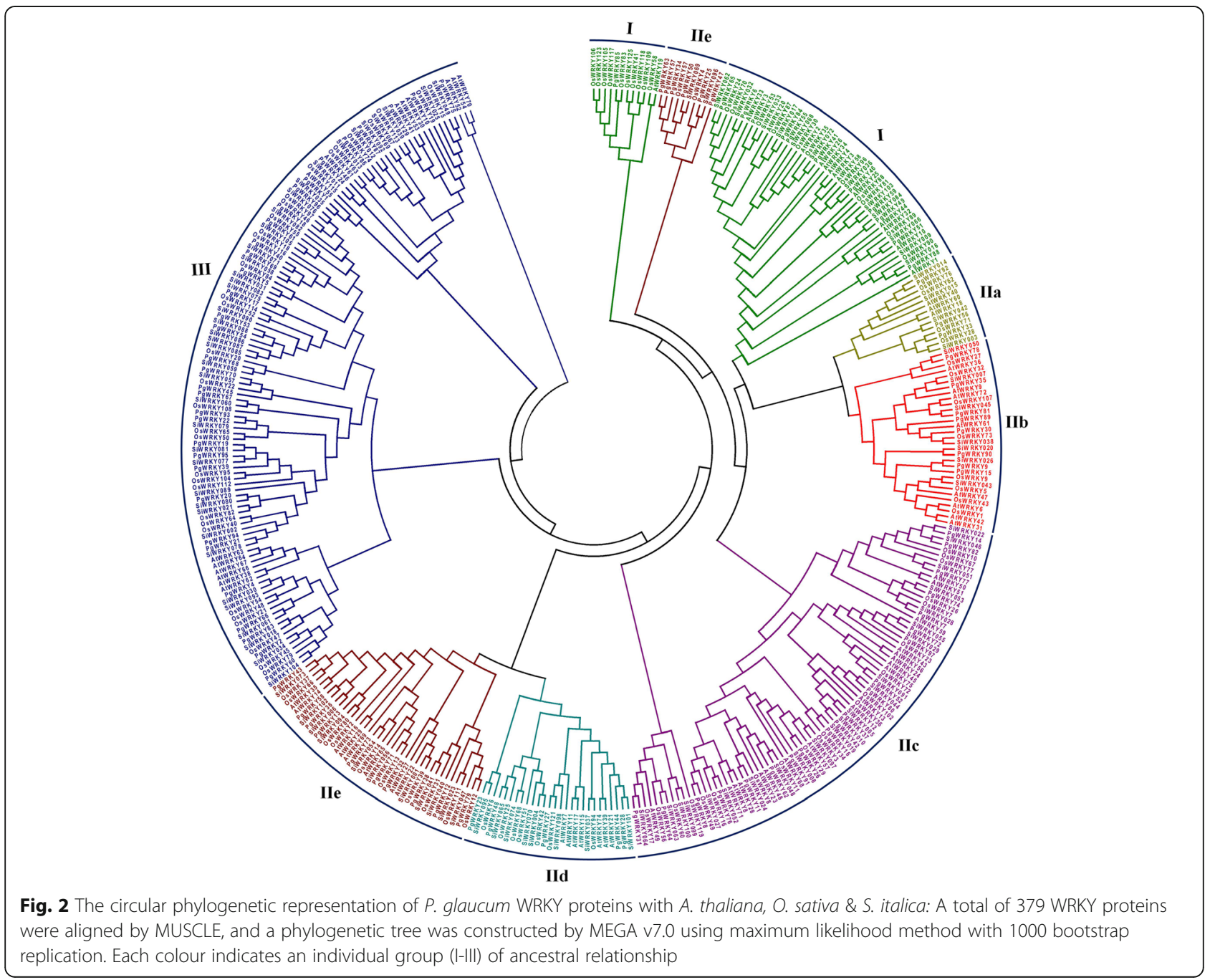




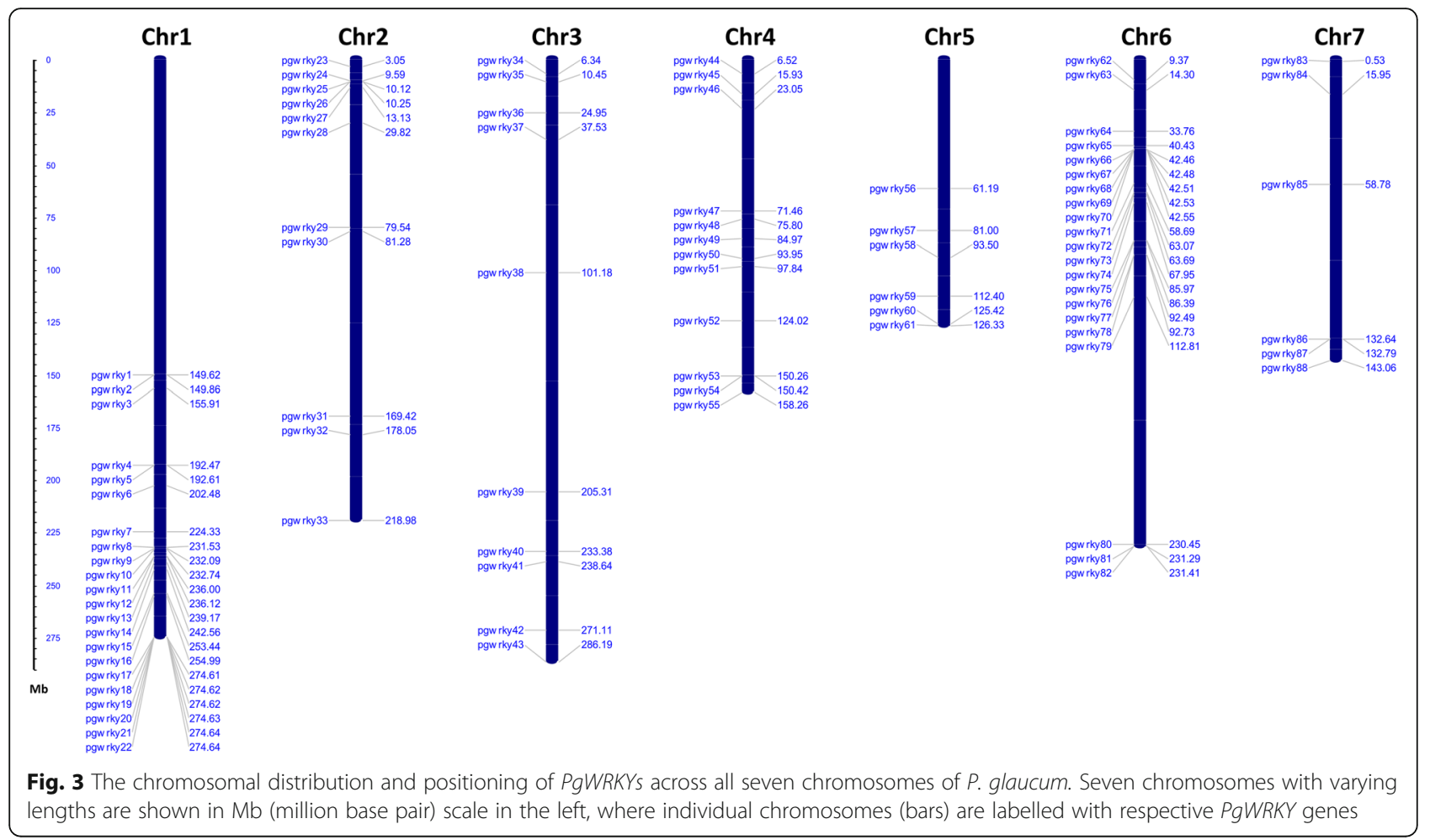

where Group I and IV members were not present respectively (Additional file 3; Figure S1).

The structural features of identified PgWRKY genes were examined in detail using the GSDS server. Figure 4 showed the varying pattern of total exonic and intronic

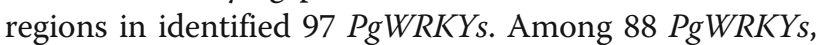
the majority of $P g W R K Y$ genes $(46.59 \%)$ had two introns and three exons; followed by $15 \operatorname{PgWRKYs}$ with one intron and two exons; $17 \operatorname{Pg} W R K Y s$ with three introns and four exons; 7 PgWRKYs with four introns and five exons; $3 \mathrm{PgWRKYs}$ with five introns and six exons; $2 \mathrm{PgWRKYs}$ with six introns and seven exons; $1 \operatorname{PgWRKY}$ with seven introns and eight exons; $1 P g W R K Y$ with sixteen introns and seventeen exons. However, PgWRKY47 had no introns (Additional file 1). We also observed variation in gene size of identified $\operatorname{Pg} W R K Y s$, which was ranging from $476 \mathrm{bp}$ (PgWRKY47) to 10,991 bp (PgWRKY26).

Further, the motif analysis was performed to identify the conserved motifs present in PgWRKYs using the MEME suite. Schematic presentation of motifs (Fig. 5) revealed that PgWRKYs contain different types of conserved motifs. We identified ten conserved motifs and named them as motif 1 to motif 10 in 97 PgWRKYs. Motif 1 (WRKY motif) was widely distributed in all members of PgWRKY family and motif 8 (WRKY motif) was only present in Group I members. We also observed group-wise specific motif conservation, i.e., motif 4 was found only in Group I members. Similarly, motif 3 was found to be present only in Group III members. We observed Group II members have a different motif distribution pattern according to subgroups (IIa-IIe), such as motif 2 was specific in Group IIa and IIb; motif 7 in Group IIb; motif 5 in Group IIc and motif 6 in Group IId members. We did not find any conserved motif in Group IIe. Group IV members did not possess any specific motif; however, motif 2 , motif 7 and motif 5 were partially conserved in few members of Group IV (Additional file 4).

\section{Synteny relationship and selection pressure analysis of WRKY orthologous genes}

Additionally, we attempted to identify the duplication event and analyzed the synteny relationship among the WRKYs of $P$. glaucum, A. thaliana, $O$. sativa and $S$. italica. A total number of 33 chromosomes ( $P$. glaucum- 7, A. thaliana- 5, O. sativa-12, S. italica- 9) with a total number of 370 WRKYs (P. glaucum- 88, A. thaliana-72, O. sativa-105, S. italica- 105) were used to map the synteny relationships. In Fig. 6, the WRKYs that were involved in segmental duplication and orthologous events were presented by different coloured lines. PgWRKYs from Chromosome 1 (PG1) and Chromosome 6 (PG6) having orthologous pairs with AT1, AT4, AT5 (A. thaliana); SI3, SI5 (S. italica) and OS1, OS5 (O. sativa) chromosomes, indicating hot-spots of PgWRKYs distribution. A total number of 10 pairs were tandemly duplicated and 13 pairs were segmentally duplicated (Additional file 5). We found 97 orthologous pairs of 


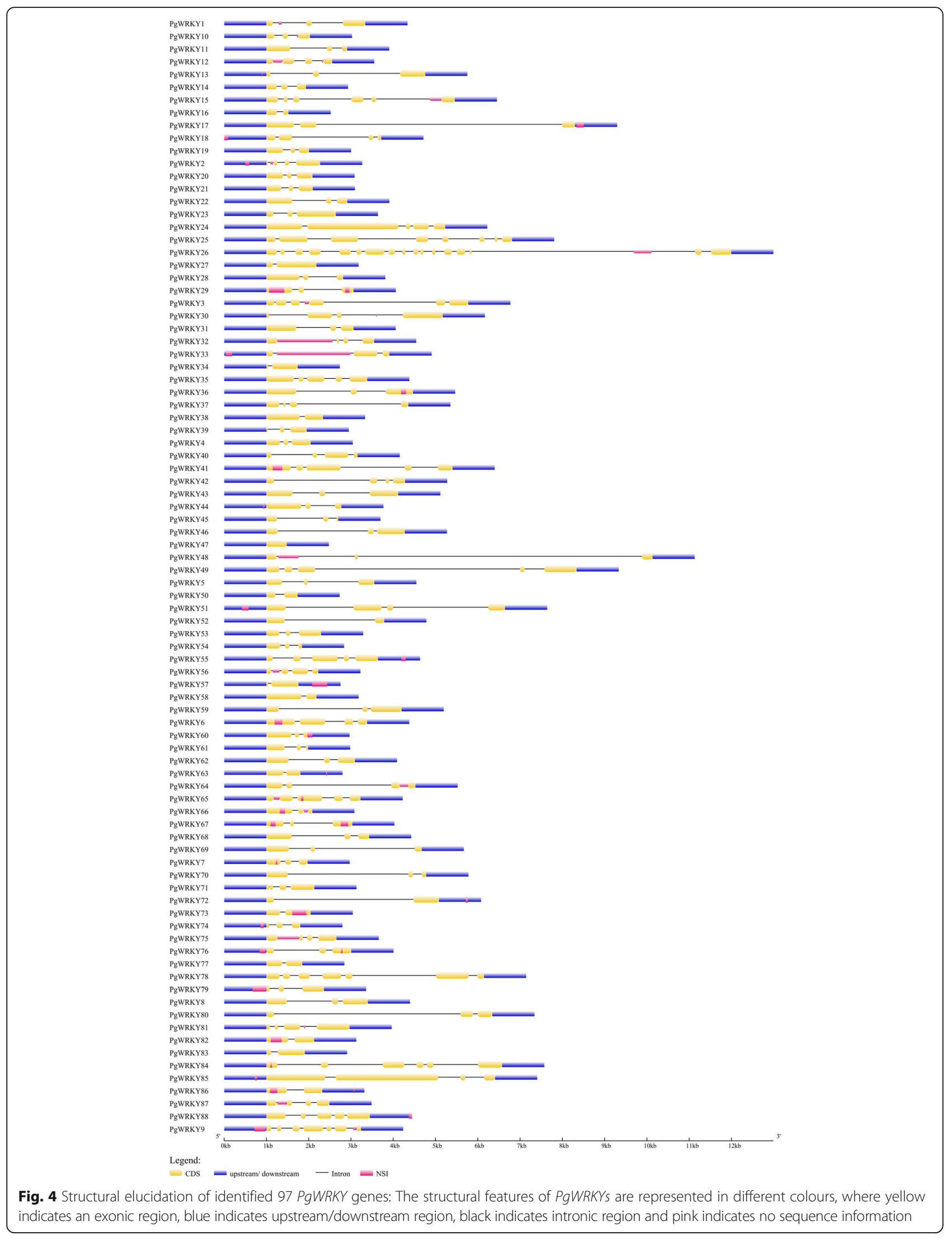




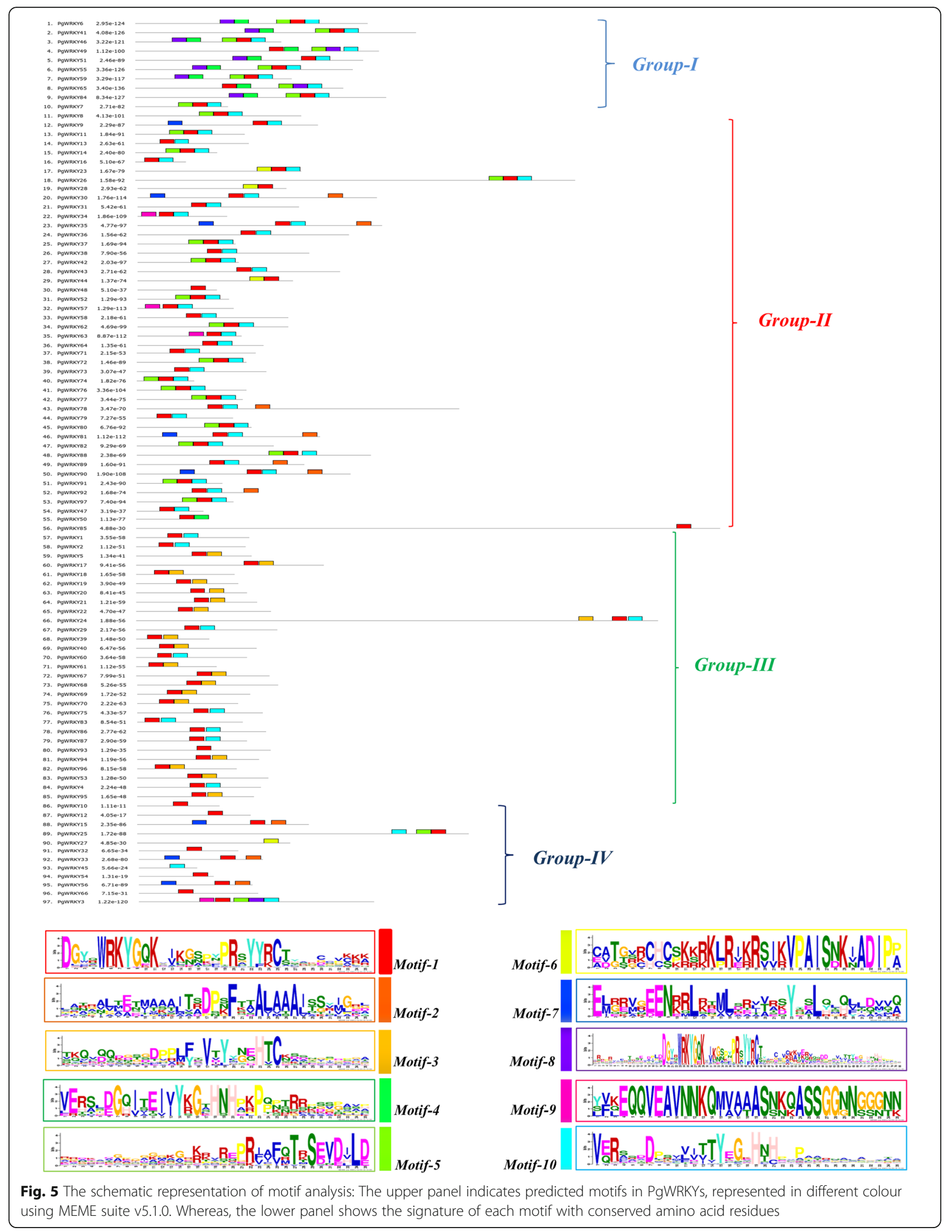




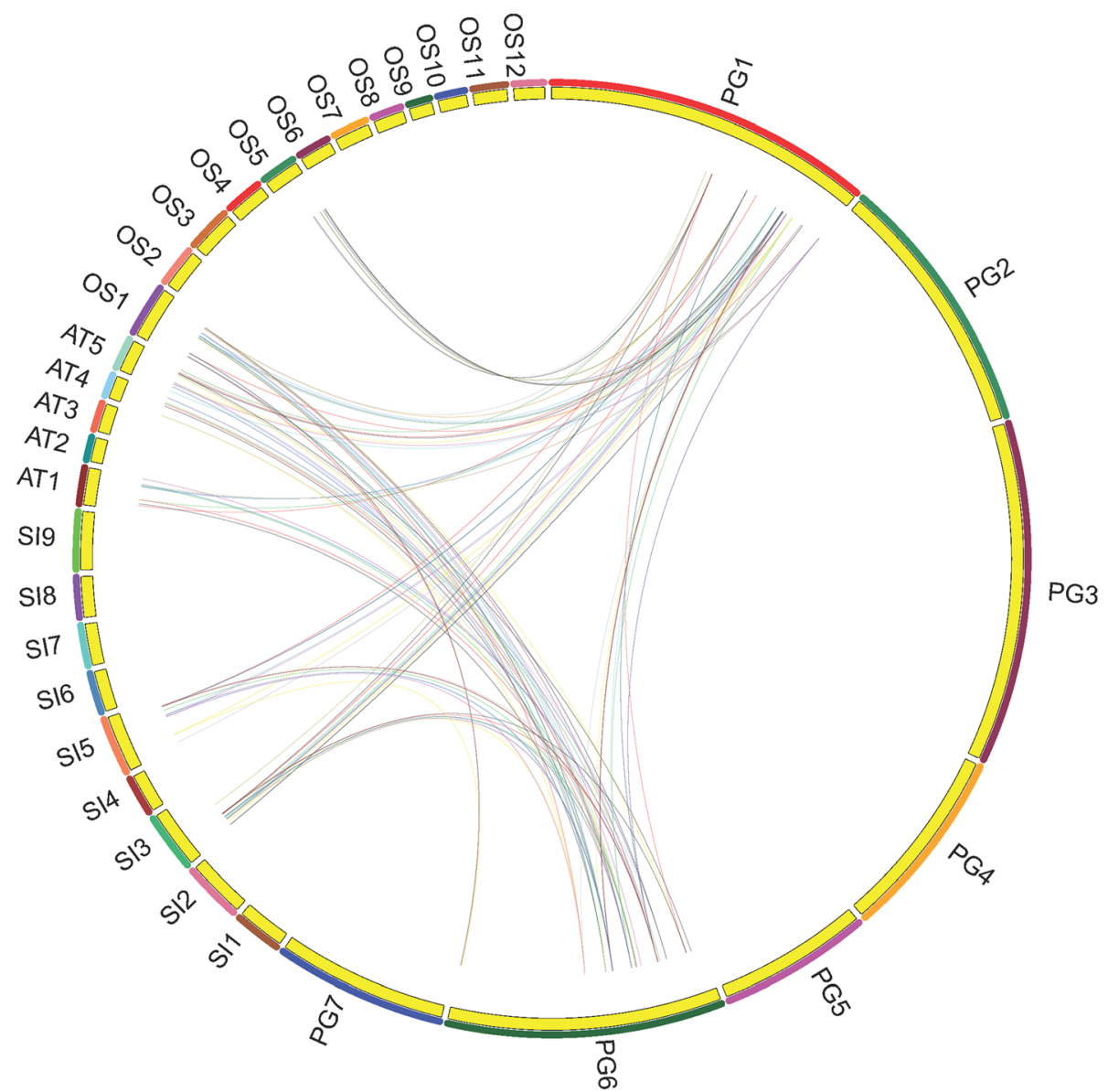

Fig. 6 The Circos plot representation for synteny relationship between P. glaucum, A. thaliana, O. sativa and S. italica WRKY genes: The synteny analysis of PgWRKYs were mapped in a circular form using CIRCOS v0.52. Each coloured curve indicates the orthologous positions of PgWRKYs across the chromosomes

PgWRKYs among WRKYs of A. thaliana, O. sativa and S. italica (Additional file 6). The Ks/Ka ratio of all identified collinear pairs was less than 1, indicating synonymous substitution or purifying selection of PgWRKYs during evolution (Additional file 7).

\section{Gene ontology annotation and cis-regulatory elements analysis}

Gene ontology (GO) annotations of 97 PgWRKY proteins were predicted using protein blast in Blast2GO tool. Based on identified GO terms, the involvement of identified PgWRKY proteins in various biological processes, cellular components and molecular functions were shown in Fig. 7. A majority of biological processes were predicted to be involved in different metabolic pathways and response to stress conditions. The molecular functions of these proteins predicted to be involved in sequence specific DNA-binding transcriptional activity. The promoter analysis of $\operatorname{Pg} W R K Y$ genes was done using PlantCARE database by taking $1.5 \mathrm{~kb}$ upstream region. A total number of 127 cis-regulatory elements (CREs) were identified among PgWRKY genes
(Additional file 8). These cis-elements in PgWRKYs were found to be specific to abiotic stress (ABRE, ARE, DRE, HSE, LTR, MBS, ACE, AE-Box, MNF1, MRE, SP1, etc.); biotic stress (EIRE, ELI-Box 3, BoxW1, WUN motif); hormonal; physiological and plant development process (AUX RR, CE1/3, GCN4 motif, SARE, MBS-I/II, MSA like, SKN motif, AS1/2, dOCT). Presence of such versatile ciselements reflecting the functional divergence of $\operatorname{PgWRKYS}$ in P. glaucum (Fig. 8).

\section{Relative expression analysis of PgWRKYs}

WRKY transcription factors are well-known for their regulatory function in various stress signaling pathways. Twenty-five $\operatorname{PgWRKY}$ genes were selected based on their sequence similarity, blast analysis, motif conservation, synteny and phylogenetic relationship with well-characterized WRKY genes of other species that are shown to be involved in abiotic stress tolerance (Additional file 9). These selected genes were subjected to transcript abundance analysis using $\mathrm{qRT}$-PCR to check their relative expression in different tissues and their probable involvement in 


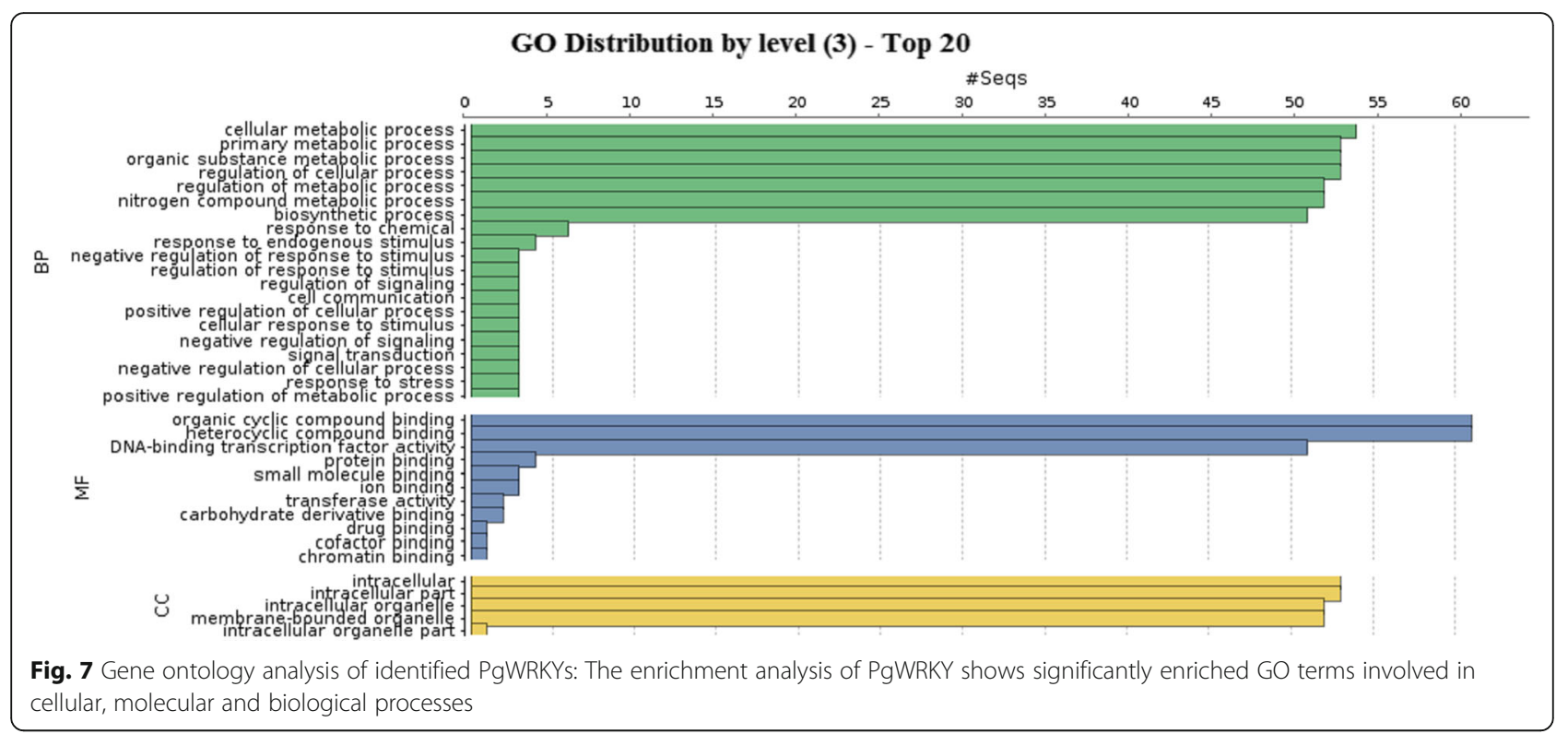

dehydration and salinity stresses. PCR conditions for these PgWRKY genes were standardized by using PgWRKY specific primers (Additional file 10) with genomic DNA of pearl millet as a template (Additional file 11; Figure S2).

Tissue specific expression analysis was performed in leaf, stem and root tissues of pearl millet. Expression analysis showed that $22 \operatorname{PgWRKYS}$ were expressed in at least one of the selected tissues (Fig. 9, Additional file 12; Figure S3).
While PgWRKY16, PgWRKY39 and PgWRKY55 were not expressed in any of the analyzed tissues. PgWRKY4, PgWRKY18 and PgWRKY96 were predominantly expressed only in root tissues. Moreover, the majority of the PgWRKYs were showing less expression in stem compared to leaf and root tissues, except PgWRKY41 and PgWRKY44. Additionally, 8 PgWRKYs (PgWRKY2, PgWRKY3, PgWRKY28, PgWRKY46,

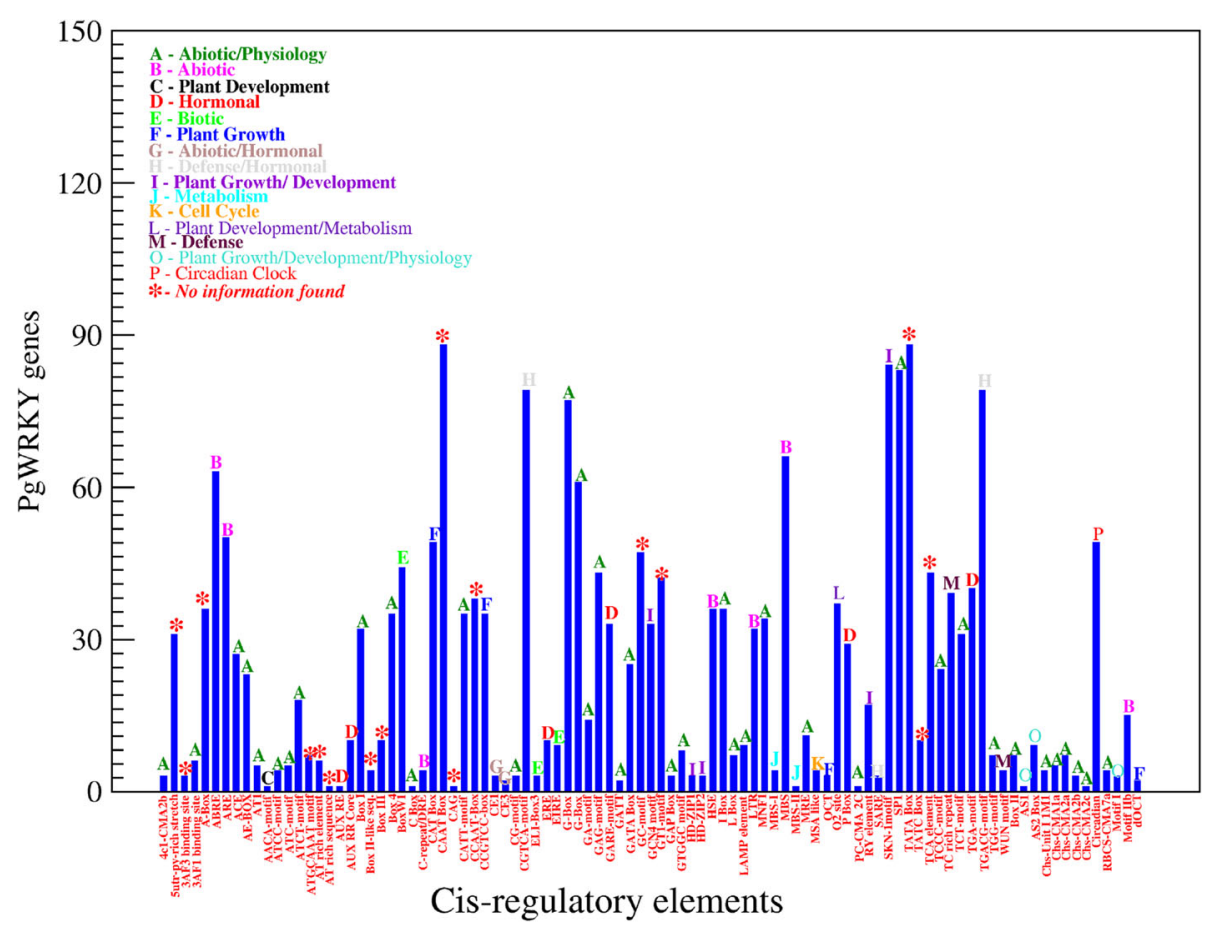

Fig. 8 In-silico analysis of Cis-regulatory elements: The frequency of identified putative cis-acting elements in the $1.5 \mathrm{~kb}$ upstream region of PgWRKY genes. The letter on each bar indicates the class (shown in legend) it belongs 


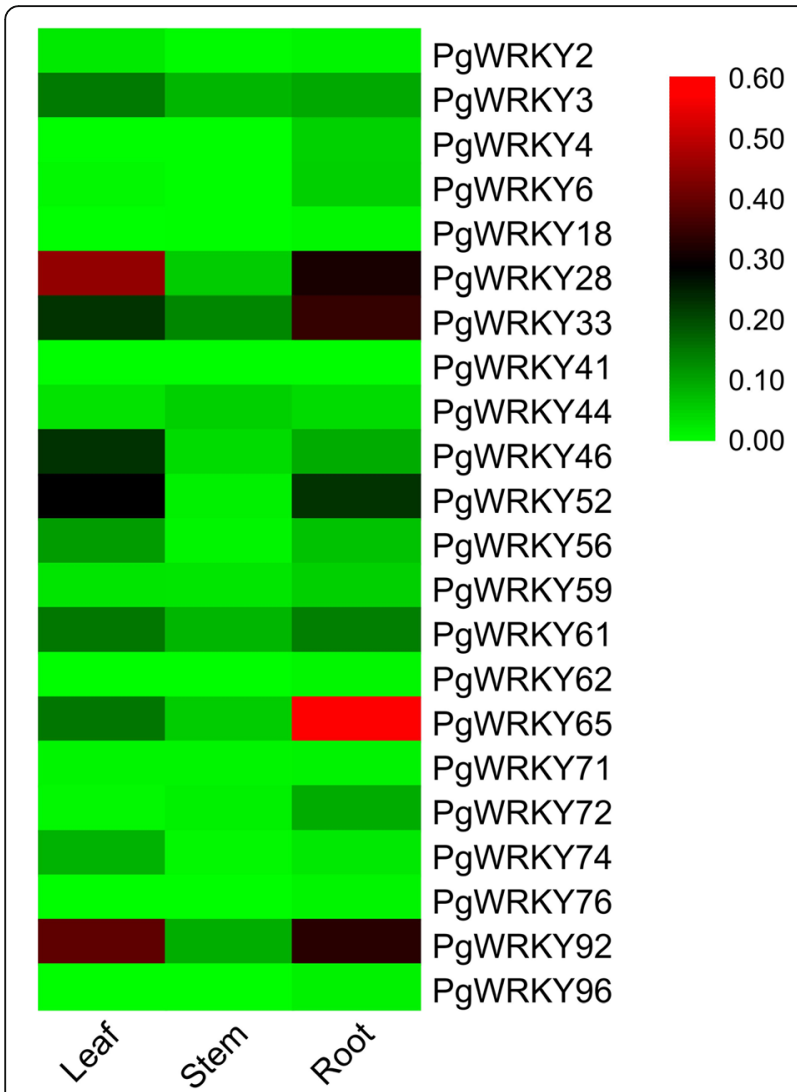

Fig. 9 Tissue specific expression analysis. Heat map showing the differential expression level of selected PgWRKYs in leaves, stem and root tissues of pearl millet. The transcript abundance level has been normalized and hierarchically clustered. Block with colours indicate decreased (green) or increased (red) transcript accumulation among the analyzed tissues. The heat map was generated using TBtools v0.66831 [37]

PgWRKY52, PgWRKY56, PgWRKY74 and PgWRKY92) showed relatively higher expression in leaves compared to stem and root; 10 PgWRKYs (PgWRKY4, PgWRKY6, PgWRKY18, PgWRKY33, PgWRKY59, PgWRKY62, PgWRKY65, PgWRKY72, PgWRKY76 and PgWRKY96) showed relatively higher expression in roots compared to leaf and stem; 2 PgWRKYs (PgWRKY41 and PgWRKY44) showed relatively higher expression in stem compared to leaf and root tissues, while remaining 2 PgWRKYs showed similar expression pattern.

The expression patterns of selected PgWRKYs were analyzed under drought and salt stress conditions at different time points using qRT-PCR. As shown in Fig. 10a and b, most of the PgWRKYs showed differential expression levels under both dehydration and salinity stress conditions at different time points. Specifically, under drought stress condition, we observed the upregulation of six PgWRKYs and downregulation of nine PgWRKYs in terms of their transcript abundance. We found the expression level of PgWRKY96 and PgWRKY61 were significantly induced under drought stress condition. Whereas PgWRKY2, PgWRKY6, PgWRKY52, and $P g W R K Y 74$ were significantly downregulated. Similarly, under salt stress condition five $\operatorname{Pg} W R K Y S$ were upregulated and nine $\operatorname{PgWRKYs}$ were down regulated when compared to control samples. The salt treated plants showed significant upregulation of $P g W R K Y 62$ and downregulation of $\operatorname{Pg} W R K Y 33, \operatorname{Pg} W R K Y 44, \operatorname{Pg} W R K Y 59$, PgWRKY61 and PgWRKY65, compared to control plants at respective time points. The transcript abundance profile of PgWRKY62 showed similar upregulation pattern in both drought and salt stress conditions. Likewise, the expression pattern of PgWRKY33 and PgWRKY65 was found to be downregulated in both drought and salt stress conditions. In addition, we could not detect the transcripts of PgWRKY4,PgWRKY16, PgWRKY39 and $P g W R K Y 55$ in both semi-quantitative as well as in quantitative RT-PCR analysis under drought and salt stress treatments.

\section{Discussion}

Pearl millet (Pennisetum glaucum) is an important member of the C4 grass family and it is cultivated in marginal lands with inadequate irrigation and resources. Regardless of its importance as a promising crop, its genomic resources like transcription factor families that play vital roles in gene regulation under stress conditions are not well studied yet. Among the plant TFs families, WRKY TFs family is one of the largest and important family with a broad range of functions in controlling plant growth, development, signal transduction and stress responses. Owing to its importance, the genome-wide identification of WRKY TFs has been carried out in pearl millet.

In this study, we have reported a total number of 97 PgWRKYs (PgWRKY1 to PgWRKY97) by complete genome screening of $P$. glaucum. These were categorized into different groups (I, II, III, IV) and subgroups (IIa, IIb, IIc, IId, IIe) based on the presence of conserved WRKY domain and zinc-finger motif like structure. Group IV members are devoid of an intact zinc-finger motif. We assume the loss of zinc-finger motif could modify the functional properties of these WRKY genes. However, it is still unclear whether the absence and variants of this zinc-finger motif affect the function and expression of WRKY genes [38]. In the course of evolution, variability in the $\mathrm{N}$ terminal domain sequence leads to the generation of new WRKY structures or different isoforms of WRKYs [36]. The WRKY signature motif variants were reported in many crop plants like rice, wheat, maize, soybean and watermelon [38] etc. Similarly, we also found substitutions and variations in amino acid residues of the signature motif of PgWRKYs. Such variants of WRKY motif can amend DNA binding activity 

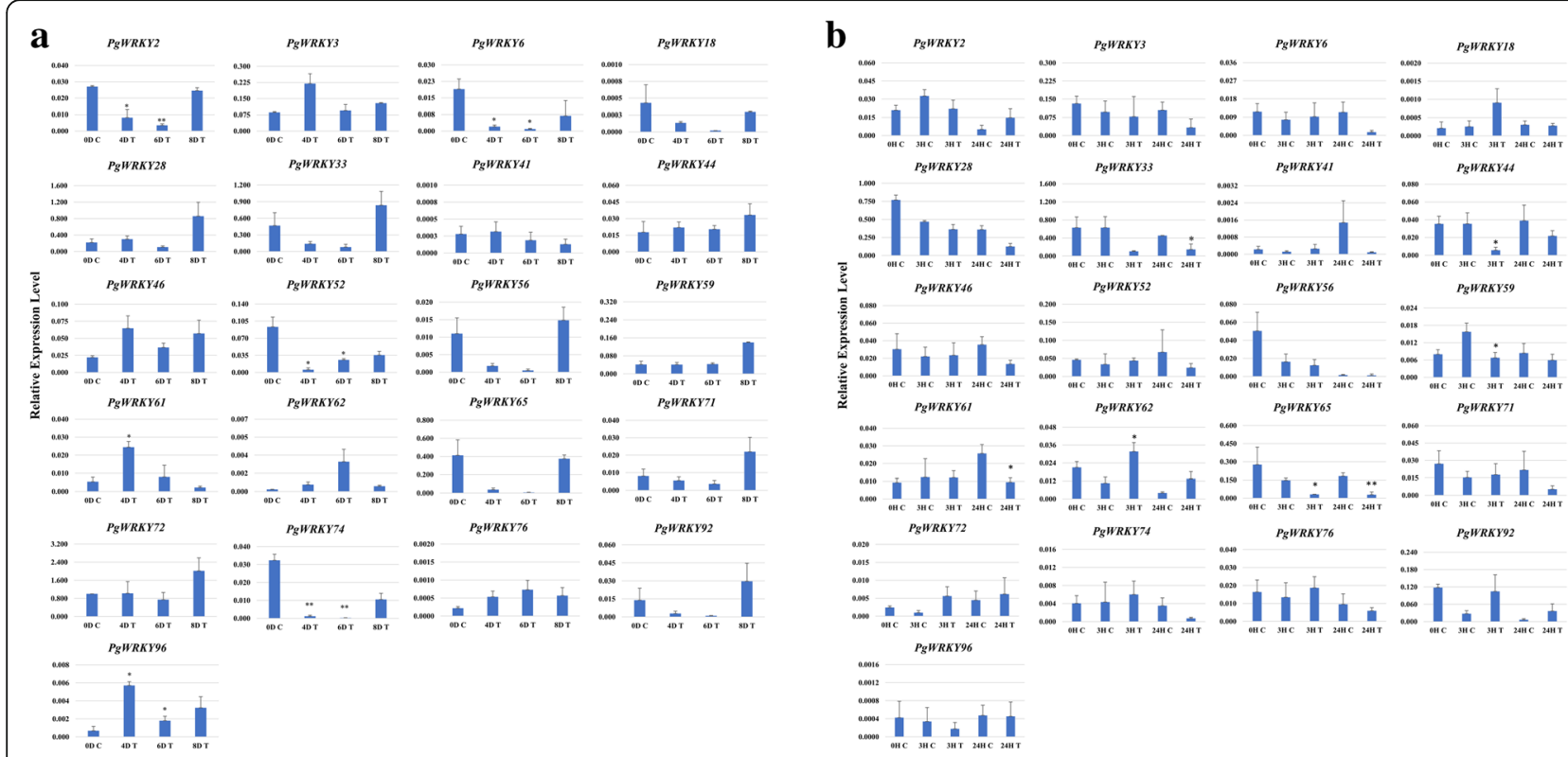

Fig. 10 Expression analysis of PgWRKYs under different abiotic stress conditions determined by qRT-PCR. The Y-axis indicates relative expression level and the $X$-axis represents different time points of stress treatment taken for expression analysis. a). Differential expression level of PgWRKYs in response to drought stress at 0th day, 4th day, 6th day and 8th day time points. b). Expression pattern of PgWRKYs under salt stress at 0-h, 3-h and 24-h time points. Each data point represents mean \pm standard deviation (SD) $(n=3)$. Significant difference in mean between the samples for a given set is indicated by ${ }^{*} P<0.05,{ }^{* *} P<0.01$, as obtained by Student's t-test

that could lead to functional diversification of WRKY genes [39]. In this context, the functional binding specificities of these PgWRKYs can be investigated extensively as future prospects of this study.

Phylogenetic analysis and clade formation support group-wise (I, II and III) classification of PgWRKYs. The close phylogenetic relationship was shown by subgroups IIa and IIb, likewise IId and IIe. It suggests the evolution of these subgroups occurred collaterally from a common ancestor. Interestingly, 12 PgWRKYs from an uncharacterized group (Group IV) were sharing clade with Group I, II and III members. This signifies that Group IV members could be evolved from these three group (I, II and III) members in the course of evolution. For example, PgWRKY3 (a Group IV member) has two WRKY domains with no zinc-finger motif at $\mathrm{N}$-terminal that clustered with Group I members. It clearly suggests that it may be evolved from Group I WRKY family of $P$. glaucum [39, 40]. The phylogenetic tree analysis, based on sequence similarity with functionally characterized WRKYs of A. thaliana, O. sativa and S. italica allowed for predicting and conferring the possible involvement of PgWRKYs in stress responsive processes.

The differential distribution pattern of $\operatorname{Pg} W R K Y s$ across the pearl millet genome implies that some chromosomal rearrangements and duplication events might have taken place in the course of evolution. The considerable variation in size, number of introns and exons in PgWRKYs suggests the loss and gain of genomic regions occurred during expansion of WRKY gene family [41]. In continuation, motif conservation analysis revealed the abundance of different type of motifs in various group members of WRKY family. Predominantly, the WRKY motif was present in all PgWRKYs, whereas some additional specific structural motifs were conserved in few PgWRKY sequences. Such structural stress-specific motifs may manifest functional specificity of these PgWRKYs under various abiotic and biotic stress conditions.

Gene duplication events are significant in the expansion and evolutionary progress of gene families [42]. Comparative mapping established the orthologous and paralogous relationship (Synteny) among dicotyledonous (Arabidopsis), and monocotyledonous (Rice, foxtail millet, pearl millet) plants. Thirty-Six PgWRKYs $(\sim 41 \%)$ were exhibited the synteny relationship with A. thaliana, $O$. sativa and S. italica. Interestingly, the presence of 13 collinear pairs between P. glaucum and S. italica, 14 collinear pairs between $P$. glaucum and $O$. sativa but not in between $P$. glaucum and A. thaliana, may suggest that these orthologous pairs were formed after the divergence of dicot and monocot plants. Similarly, the existence of 10 collinear pairs between $P$. glaucum and A. thaliana but not in between $P$. glaucum, $O$. sativa and S. italica, may suggest that these orthologous pairs may be involved in divergence of dicot and monocot plants. Additionally, 11 PgWRKYs were collinearly paired with WRKYs of $A$. thaliana, $O$. sativa and $S$. italica, which indicates that these orthologous pairs may already exist before the 
ancestral divergence [43]. The functional information of PgWRKYs can be predicted according to their identified orthologous WRKYs in Arabidopsis, Rice and Foxtail millet. Synteny relationship indicates that some PgWRKY genes were possibly generated by gene duplication and rearrangement events. The Ks/Ka ratio of identified collinear pairs emphasizes more on negative pressure selection according to Darwin's natural selection process for amino acid conservation. The comparison study revealed that there was a higher number of orthologs pair with respect to paralogs. This shows that orthologs evolve with a higher evolutionary rate unlike paralogs [44].

Identified PgWRKYs conserved with various types of cis-regulatory elements in their promoter regions, suggesting that these $P g W R K Y s$ could be involved in different biological processes associated with plant growth and development [32]. The presence of various abiotic stress-specific cis-regulatory elements (Fig. 8) in the promoter region of identified PgWRKYs may be associated in providing natural tolerance to $P$. glaucum. Furthermore, the presence of various cis-regulatory elements responding to phytohormones (ABA, MeJA and SA), indicate their involvement in controlling various hormonal signaling pathways linked with abiotic and biotic stress management in P. glaucum.

Selected twenty-five PgWRKYs for expression analysis were representing all four groups (I, II, III, IV) and more or less evenly distributed throughout the genome except chromosome 7 of P. glaucum. These PgWRKYs showed differential expression levels in leaf, stem and root tissues. Most of the selected PgWRKYs showed higher expression in leaves and root as compared to the stem tissues. PgWRKY4, PgWRKY18 and PgWRKY96 were found to be expressed predominantly in root tissue. Similarly, transcripts of PgWRKY2, PgWRKY46 and $P g W R K Y 74$ were most abundant in leaf compared to root and stem tissues. This suggests that these PgWRKYs might be related to tissue specific development and signaling processes in P. glaucum. Interestingly, transcripts of PgWRKY16, PgWRKY39 and PgWRKY55 (belonging to Group III) could not be detected in any of these tissues; implying that these PgWRKYs could be involved in other physiological processes of $P$. glaucum. Previous studies also indicated that WRKY genes participate in transcriptional regulation of downstream target genes that are involved in various physiological and developmental pathways $[20,25]$.

The WRKY gene family is significantly involved in regulation of plant responses to various abiotic and biotic stresses [20, 45-48]. PgWRKYs exhibited differential expression patterns at different time points of dehydration

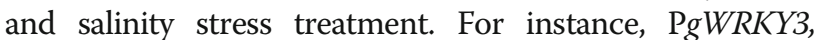
$P g W R K Y 46, P g W R K Y 61$ and PgWRKY96 were upregulated while $P g W R K Y 52$ was downregulated at 4th day time point under dehydration stress. Similarly, in salt stress condition, transcripts of PgWRKY18 and PgWRKY72 were enhanced after $3 \mathrm{~h}$ (early time point) and $\operatorname{PgWRKY2}$ at $24 \mathrm{~h}$ (late time point) post treatment. In addition, few $\operatorname{Pg} W R K Y s$ were remarkably suppressed, including $\operatorname{Pg} W R K Y 44$ and $P g W R K Y 59$ at early time point and $P g W R K Y 3$, PgWRKY6, PgWRKY46, PgWRKY61 and PgWRKY74 at late time point of $\mathrm{NaCl}$ treatment. Such differential expression of $\operatorname{PgWRKYs}$ under stress conditions at different time points suggest their intricate response/ $s$ in the regulatory network of plant abiotic stress processes.

The PgWRKY2, PgWRKY6, PgWRKY61, PgWRKY62, $P g W R K Y 65$ and $P g W R K Y 74$ were significantly expressed under dehydration stress. Interestingly, the transcript abundance of these genes on 8th day (after recovery) post treatment was comparable with zero-day (control) samples, which strongly indicates the involvement of these $P g W R K Y s$ in drought stress response (Additional files 13 and 14; Figure S4). The expression pattern PgWRKY62, PgWRKY33,PgWRKY44,PgWRKY59, PgWRKY61 and $P g W R K Y 65$ indicated their involvement in salinity stress responses of pearl millet.

The expression patterns of PgWRKY62, PgWRKY33 and PgWRKY65 suggested their involvement in both drought and salt stress responses. Interestingly, the ortholog of PgWRKY62 in rice (OsWRKY11) was also shown to be involved in multiple abiotic stresses [49]. Previous studies also showed that individual transcription factors might be involved in multiple signaling pathways. For example, AtWRKY39 involved in both heat stress and hormone signaling [47]. Interestingly, W-box element (binding site for WRKY TF) was located in upstream region of PgWRKY62 and PgWRKY33, which suggests that these PgWRKY genes might auto-regulate their expression during stress conditions. Taken together, in-silico analysis of identified PgWRKYs and their transcriptional profiling would help in candidate $\operatorname{Pg} W R K Y$ genes selection for delineating their functional roles in abiotic stress tolerance mechanism of pearl millet.

\section{Conclusions}

The research reported in the manuscript describe the genome wide identification of WRKY TFs and their transcriptional profiling in response to dehydration and salinity stress. Furthermore, our findings provide the foundation for further functional characterization and identification of the regulatory mechanism of $\operatorname{Pg} W R K Y s$ in plant stress responses. Additionally, candidate PgWRKYs can be employed for crop improvement using molecular breeding techniques and genome editing tools to enhance agricultural production for ensuring future food security. 


\section{Methods}

In-silico database mining for the identification of WRKY proteins from $P$. glaucum

The protein sequences containing WRKY domain of Arabidopsis thaliana, Oryza sativa (rice), and Setaria italica (foxtail millet) were used as reference sequences for the identification of WRKY proteins in P. glaucum. A total of 38,579 proteome sequences of $P$. glaucum were retrieved from pearl millet genome database (http://cegsb.icrisat.org/ipmgsc/) [50] and a total of 282 reference sequences (Additional file 15) were downloaded from the Phytozome v12.1.6, Plant Genomics Resource, The Arabidopsis Information Resource (TAIR) ((https://www.arabidopsis.org/) and Oryzabase; An Integrated Biological and Genome Information Database for Rice [51-53]. The search for WRKY protein sequences in $P$. glaucum was initiated with the construction of HMM profile. The approach uses a Position-Specific Scoring System as well as the Hidden Markov Model to generate a HMM profile using HMMER tool v3.2 with default parameters [54] based on the degree of conservation from multiple sequence alignment. Initially, the reference sequences were aligned using Clustal omega [55] and the HMM profile was build based on the obtained consensus of the aligned sequences. By using the generated WRKY profile, the HMMER search was performed against the proteome database to identify probable homologous WRKY sequences in P. glaucum. Furthermore, the HMMER scan was performed to confirm the presence of WRKY domain in identified putative WRKY homologs using the PFAM domain (PF03106) [56].

\section{Phylogenetic tree construction and sequence analysis}

The WRKY protein sequences of P. glaucum, A. thaliana, O. sativa and S. italica and were aligned using MUSCLE with default parameters [57] and imported into the MEGA v7.0 software [58] to construct an evolutionary relationship tree using Maximum likelihood method. The parameters used for the phylogenetic tree construction are bootstrap test method for 1000 replications; Substitution model: Jones-Taylor-Thronton (JTT) model; Rates among sites: gamma distributed (G) and Gaps/Missing Data Treatment: Partial deletion. Each WRKY protein sequence of P. glaucum was analyzed for their physiochemical characteristics such as the number of amino acids, molecular weight, isoelectric point, instability index, aliphatic index and hydropathicity using ProtParam tool on the ExPASy server [59].

\section{Chromosomal mapping and gene structure}

To visualize the chromosomal position of each WRKY gene, the chromosomal coordinates with starting and ending points in ascending order were imported into MapInspect v1.0 software (http://mapinspect.software. informer.com/). The physical map of the WRKY genes was constructed by locating the genes on each of the seven chromosomes. Coding sequences of identified WRKYs were retrieved from pearl millet genome database (http://cegsb.icrisat.org/ipmgsc/) [50] and genomic sequences of each WRKY were extracted using available genomic coordinates. The intronic and exonic positions of all WRKY genes in P. glaucum were predicted using the coding and genomic sequences in GSDS web server v2.0 [60]. Further, these WRKYs were analyzed for conserved motifs using the MEME suite v5.1.0 [61].

\section{Collinearity mapping and the calculation of synonymous} (Ks) \& non-synonymous $\mathrm{Ka}$ ) ratio (Ks/Ka)

$P$. glaucum WRKY sequences were searched for sequence homologs against the WRKY families of $A$. thaliana, O. sativa (rice) and S. italica (foxtail millet), using BlastP (e-value cut-off 0.001) analysis. The MCScanX v0.8 software [62] was used to identify the orthologous pairs, tandem and segmental duplications among WRKY genes. The synteny map of orthologous and paralogous pairs across the species was shown in Circos plot generated by using $\mathrm{R}$ software [63]. Further, the substitution rates including the synonymous (Ks) and non-synonymous (Ka) ratio for the potential collinear pairs were estimated using the PAL2NAL server v14.0 [64] by providing pairwise sequence alignment information and the coding sequences of collinear pair. To achieve this, the EMBOSS Water algorithm was used for the pairwise alignment to identify the similar local regions in the collinear pairs and the coding sequence information was downloaded from pearl millet genome database.

\section{Gene ontology annotation and identification of cis- regulatory elements}

The gene ontology (GO) terms for the WRKY proteins were identified using default parameters in Blast2GO v5 tool [65]. Initially, the sequences were screened using BLASTP, followed by mapping, InterProScan analysis and annotation. Furthermore, the biological processes, cellular components and metabolic pathways were predicted using identified GO terms. We have also analyzed the promoter region for identifying the presence of cisregulatory elements by taking $1500 \mathrm{bp}$ upstream region of all pearl millet WRKY genes using the Plant CARE database [66].

\section{Plants material and stress treatment}

Pearl millet germplasm (Preservation Accession number: IP 3757, ISP number: 2347, Collector number: SAR 1148) [67] obtained from Gene bank, International Crops Research Institute for Semi-Arid Tropics (ICRISAT, an Institute of CGIAR with large repository of pearl millet germplasm), Patancheru, India through a 
material transfer agreement (MTA). Seeds of IP 3757 were sown in pots, filled with a mixture of soil and vermiculture (at a ratio of $1: 1, \mathrm{v} / \mathrm{v}$ ), and then grown in greenhouse conditions having a temperature $25^{\circ} \mathrm{C}( \pm 2)$, photoperiod of $16 \mathrm{~h} \mathrm{light/} 8 \mathrm{~h}$ dark.

The relative expression level of WRKY genes were studied in the selected cultivar in different tissues and under drought and salinity stress conditions at different time points. The leaves, stem and root tissues were harvested under normal growth conditions from four-week-old seedlings of pearl millet. Drought condition was created by withdrawing irrigation for 6 days after 4th week of sowing of pearl millet seeds and the control plants were regularly watered on alternate days. On 7th day, irrigation was done for both control and treated plants for their recovery. The leaves of both control and treated plants were collected at day 0, 4, 6 and 8 respectively [68]. For salt stress, four-week-old seedlings were washed and immersed in $250 \mathrm{mM} \mathrm{NaCl}$ solution while for control treatment, seedlings were kept in distilled water. Leaf samples were collected from both control and treated plants at time points of $0 \mathrm{~h}, 3 \mathrm{~h}$ (early) and $24 \mathrm{~h}$ (late) [29]. At each time point, the leaves of three different plants were harvested as a single sample and immediately frozen in liquid nitrogen and stored at $-80^{\circ} \mathrm{C}$ until further analysis.

\section{Relative expression level of WRKY genes}

Phylogeny, homologous pairing, sequence similarity and blast results of all WRKY genes of pearl millet were analyzed with well characterized WRKY genes of A. thaliana, $O$. sativa and S. italica and in response to various abiotic stresses for selection of candidate WRKYs genes of pearl millet. The transcript level of these selected WRKYs were analysed to check their differential expression pattern. Primers were designed for selected WRKYs using Primer3 input [69] (http://primer3.ut.ee/), according to the following criteria: i) amplicon size of $100-150 \mathrm{bp}$; ii) primer length of 18-23 bases; iii) melting temperature of 57$63{ }^{\circ} \mathrm{C}$ and iv) GC content of $40-60 \%$. The PCR conditions were standardized for each pair of primers using the genomic DNA of pearl millet as a template.

Total RNA was extracted from frozen plant tissues using RNA extraction kit (STRN50, Sigma Aldrich, St. Louis, USA) following the manufacturer's protocol. RNA concentration and purity were confirmed using Nanodrop 2000 spectrophotometer (Thermo Scientific, Wilmington, DE, USA) for each sample. The integrity of extracted RNA was also ensured by resolving on $1.2 \%$ agarose gel containing $18 \%$ formaldehyde. For each sample, $1 \mu \mathrm{g}$ of total RNA was treated with RNase free DNase I (Sigma-AMPD1, St. Louis, USA) and reverse transcribed to synthesize cDNA using first strand cDNA synthesis kit (K1612, Thermo Scientific, MA, USA). Semi-quantitative RT-PCR was done for 35 cycles using prepared cDNA. The qRT-PCR was performed in ABI StepOne Real-Time PCR System (Applied Biosystems, Foster City, CA, USA). The reaction mixtures of $20 \mu \mathrm{l}$ contained $10 \mu \mathrm{l}$ of SYBR Premix buffer (Mesa green qPCR master mix-Eurogentec), $1.0 \mu \mathrm{l}$ from each forward and reverse primer $(5 \mu \mathrm{M}), 2 \mu \mathrm{l}(20 \mathrm{ng})$ of cDNA and $6 \mu \mathrm{l}$ nuclease free water. Negative control samples were having all the ingredients as mentioned above except the cDNA template. Each reaction was run in three technical and three biological replicates to obtain an average value with respective standard deviation. The amplification conditions were: $95^{\circ} \mathrm{C}$ for $10 \mathrm{~min}$ followed by 40 cycles of $95^{\circ} \mathrm{C}$ for $15 \mathrm{~s}, 58^{\circ} \mathrm{C}$ for $1 \mathrm{~min}$. Fluorescent intensity data was collected, and the reaction specificity was confirmed by melting curve analysis $\left(58^{\circ} \mathrm{C}\right.$ to $95^{\circ} \mathrm{C}$, at increments of $0.3^{\circ} \mathrm{C}$ ). The constitutively expressed $E F 1 \alpha[70]$ and GAPDH [71] genes were used as endogenous controls.

\section{Supplementary information}

Supplementary information accompanies this paper at https://doi.org/10. 1186/s12864-020-6622-0.

Additional file 1: Table S1. Protein features and chromosomal details of identified 97 PgWRKYs.

Additional file 2: Table S2. Different types of WRKY variants in four groups of PgWRKY family.

Additional file 3: Figure S1. Group-Wise distribution of PgWRKY genes among the seven chromosomes of P. glaucum.

Additional file 4: Table S3. Motif distribution across all the identified 97 PgWRKYs.

Additional file 5: Table S4. List of tandem and segmental duplicated PgWRKYs.

Additional file 6: Table S5. List of orthologous pair of $P$. glaucum with S. italica, O. sativa and A. thaliana.

Additional file 7: Table S6. The Ks/Ka ratio of identified collinear pairs. Additional file 8: Table S7. List of cis-regulatory elements (CREs) present in promoter region of identified PgWRKYS.

Additional file 9: Table S8. Selected PgWRKYs for expression analysis and their group representation with motif conservation.

Additional file 10: Table S9. List of primers used in $\mathrm{QRT}$-PCR analysis for selected PgWRKYs.

Additional file 11: Figure S2. Standardization of PCR conditions for selected PgWRKYs and endogenous control genes (GAPDH and EF1a) using pearl millet genomic DNA as a template.

Additional file 12: Figure S3. Expression level of selected PgWRKYs in different tissues (leaves, root and stem) of pearl millet.

Additional file 13: Figure S4. Influence of drought stress treatment on four-week old pearl millet seedlings. A) Growth rates of control and treated seedlings at 0th day and 6th day. B) Recovery level on 8th day of drought stressed seedlings after re-watering on 7th day.

Additional file 14: Figure S5. Salt stress treatment on four-week-old seedlings of pearl millet (IP3757). A) Control and treated seedlings at $0 \mathrm{~h}$ of treatment. B). The response of control and treated seedlings to 250 $\mathrm{mM} \mathrm{NaCl}$ at $24 \mathrm{~h}$ after treatment.

Additional file 15: Table S10. A list of accession numbers of reference WRKYs used to access the data analyzed in this study.

\section{Abbreviations}

ABA: Abscisic Acid; AtWRKY: Arabidopsis thaliana WRKY; CREs : Cis-regulatory elements; GO: Gene ontology; MeJA: Methyl Jasmonate; OsWRKY: Oryza sativa WRKY; PgWRKY: Pennisetum glaucum WRKY; SA: Salicylic acid; 
SiWRKY: Setaria italica WRKY; TaWRKY: Triticum aestivum; TFs: Transcription factors

\section{Acknowledgements}

We are thankful to ICRISAT, Hyderabad for providing the seed material. University grant commission (UGC, India) for providing fellowship to JC.

\section{Authors' contributions}

JC performed all the experiments, bioinformatics data analysis and wrote the manuscript. SS and AD performed bioinformatics analysis and participated in bioinformatics writing part. AP guided in designing experiments and arranged seeds. MKG and ND conceptualized the project, designed the experiments and participated in writing the manuscript. All authors have read and approved the manuscript.

\section{Funding}

Research on pearl millet genomics at ND's laboratory was funded by the core grant of Institute of Life Sciences, Bhubaneswar, Govt. of India. The funding bodies had no role in the design of the study, collection, analysis, or interpretation of data or in the writing of the manuscript.

\section{Availability of data and materials}

All data analyzed during this study are included in this article and its additional files.

\section{Ethics approval and consent to participate}

Not applicable.

\section{Consent for publication}

Not applicable.

\section{Competing interests}

The authors declare that they have no competing interests.

Received: 23 December 2019 Accepted: 25 February 2020 Published online: 14 March 2020

\section{References}

1. Parry M, Arnell N, McMichael T, Nicholls R, Martens P, Kovats S, Livermore M, Rosenzweig C, Iglesias A, Fischer G. Millions at risk: defining critical climate change threats and targets. Glob Environ Chang. 2001;11(3):181-3.

2. Ziervogel G, New M, Archer van Garderen E, Midgley G, Taylor A, Hamann R, Stuart-Hill S, Myers J, Warburton M. Climate change impacts and adaptation in South Africa. Wiley Interdiscip Rev Clim Chang. 2014;5(5):605-20.

3. Beddington JR, Asaduzzaman M, Fernandez A, Clark ME, Guillou M, Jahn MM, Erda L, Mamo T, Bo N, Nobre CA. Achieving food security in the face of climate change: final report from the commission on sustainable agriculture and climate change; 2012.

4. Sehgal D, Rajaram V, Armstead IP, Vadez V, Yadav YP, Hash CT, Yadav RS. Integration of gene-based markers in a pearl millet genetic map for identification of candidate genes underlying drought tolerance quantitative trait loci. BMC Plant Biol. 2012;12(1):9.

5. Vadez V, Hash T, Bidinger F, Kholova J. II.1.5 Phenotyping pearl millet for adaptation to drought. Front Physiol. 2012:3(386). https://doi.org/10.3389/ fphys.2012.00386.

6. Nambiar VS, Dhaduk J, Sareen N, Shahu T, Desai R. Potential functional implications of pearl millet (Pennisetum glaucum) in health and disease. J Appl Pharm Sci. 2011;1(10):62.

7. Tako E, Reed SM, Budiman J, Hart J, Glahn RP. Higher iron pearl millet (Pennisetum glaucum L.) provides more absorbable iron that is limited by increased polyphenolic content. Nutr J. 2015;14(1):11.

8. Singh P, Boote K, Kadiyala MDM, Nedumaran S, Gupta SK, Srinivas K, Bantilan MCS. An assessment of yield gains under climate change due to genetic modification of pearl millet. Sci Total Environ. 2017;601-602:1226-37.

9. El Sabagh A, Hossain A, Islam MS, Barutçular C, Hussain S, Hasanuzzaman M, Akram T, Mubeen M, Jatoi W, Fahad S, et al. Drought and salinity stresses in barley: consequences and mitigation strategies. Aust J Crop Sci. 2019;13: $810-20$

10. Aredo S. Famine and food security in Ethiopia: Lessons for Africa: Patrick Webb and Joachim von Braun. John Wiley, Chichester, 1994, vii + 158 pp. [UK pound]14.95. ISBN 0-471-94821-7. Agric Econ. 1995;13(1):71-2.
11. Cenacchi N. Drought risk reduction in agriculture: a review of adaptive strategies in East Africa and the indo-Gangetic plain of South Asia. IFPR/ Discussion Paper 1372, Washington. Int Food Policy Res Inst (IFPRI). 2014. http://ebrary.ifpri.org/cdm/ref/collection/p15738coll2/id/128277.

12. Shrivastava $P$, Kumar R. Soil salinity: a serious environmental issue and plant growth promoting bacteria as one of the tools for its alleviation. Saudi J Biol Sci. 2015;22(2):123-31.

13. Panta S, Flowers T, Lane P, Doyle R, Haros G, Shabala S. Halophyte agriculture: success stories. Environ Exp Bot. 2014;107:71-83.

14. Swarajyalakshmi G, Gurumurthy P, Subbaiah GV. Soil salinity in South India: problems and solutions. J Crop Prod. 2003;7(1-2):247-75.

15. Shrestha A, Khan A. Dey N: cis-trans engineering: advances and perspectives on customized transcriptional regulation in plants. Mol Plant. 2018;11(7):886-98.

16. Singh KB, Foley RC, Oñate-Sánchez L. Transcription factors in plant defense and stress responses. Curr Opin Plant Biol. 2002;5(5):430-6.

17. Agarwal PK, Agarwal P, Reddy MK, Sopory SK. Role of DREB transcription factors in abiotic and biotic stress tolerance in plants. Plant Cell Rep. 2006; 25(12):1263-74

18. Shao $\mathrm{H}$, Wang $\mathrm{H}$, Tang $X$. NAC transcription factors in plant multiple abiotic stress responses: progress and prospects. Front Plant Sci. 2015;6(902). https://doi.org/10.3389/fpls.2015.00902.

19. Sakuma Y, Liu Q, Dubouzet JG, Abe H, Shinozaki K, Yamaguchi-Shinozaki K. DNA-binding specificity of the ERF/AP2 domain of Arabidopsis DREBs, transcription factors involved in dehydration- and cold-inducible gene expression. Biochem Biophys Res Commun. 2002;290(3):998-1009.

20. Phukan UJ, Jeena GS, Shukla RK. WRKY transcription factors: molecular regulation and stress responses in plants. Front Plant Sci. 2016;7:760.

21. Ambawat $S$, Sharma P, Yadav NR, Yadav RC. MYB transcription factor genes as regulators for plant responses: an overview. Physiol Mol Biol Plants. 2013; 19(3):307-21.

22. Boter M, Ruiz-Rivero O, Abdeen A, Prat S. Conserved MYC transcription factors play a key role in jasmonate signaling both in tomato and Arabidopsis. Genes Dev. 2004;18(13):1577-91.

23. Jakoby M, Weisshaar B, Dröge-Laser W, Vicente-Carbajosa J, Tiedemann J, Kroj T, Parcy F. bZIP transcription factors in Arabidopsis. Trends Plant Sci. 2002;7(3):106-11.

24. Eulgem T, Rushton PJ, Robatzek S, Somssich IE. The WRKY superfamily of plant transcription factors. Trends Plant Sci. 2000;5(5):199-206.

25. Bakshi M, Oelmüller R. WRKY transcription factors. Plant Signal Behav. 2014; 9(2):e27700.

26. Rushton PJ, Somssich IE, Ringler P, Shen QJ. WRKY transcription factors. Trends Plant Sci. 2010;15(5):247-58.

27. Jimmy $\lrcorner$, Babu S. Variations in the structure and evolution of Rice WRKY genes in Indica and japonica genotypes and their co-expression network in mediating disease resistance. Evol Bioinformatics Online. 2019;15: 1176934319857720.

28. Dou L, Zhang X, Pang C, Song M, Wei H, Fan S, Yu S. Genome-wide analysis of the WRKY gene family in cotton. Mol Gen Genomics. 2014;289(6):1103-21.

29. Muthamilarasan M, Bonthala VS, Khandelwal R, Jaishankar J, Shweta S, Nawaz K, Prasad M. Global analysis of WRKY transcription factor superfamily in Setaria identifies potential candidates involved in abiotic stress signaling. Front Plant Sci. 2015;6:910

30. Wang M, Vannozzi A, Wang G, Liang Y-H, Tornielli GB, Zenoni S, Cavallini E, Pezzotti M, Z-MM C. Genome and transcriptome analysis of the grapevine (Vitis vinifera L.) WRKY gene family. Horticulture research. 2014;1:14016.

31. Mohanta TK, Park Y-H, Bae H. Novel genomic and evolutionary insight of WRKY transcription factors in plant lineage. Sci Rep. 2016;6:37309.

32. Goel R, Pandey A, Trivedi PK, Asif MH. Genome-wide analysis of the Musa WRKY gene family: evolution and differential expression during development and stress. Front Plant Sci. 2016;7:299.

33. Huang S, Gao Y, Liu J, Peng X, Niu X, Fei Z, Cao S, Liu Y. Genome-wide analysis of WRKY transcription factors in Solanum lycopersicum. Mol Gen Genomics. 2012;287(6):495-513.

34. Li D, Liu P, Yu J, Wang L, Dossa K, Zhang Y, Zhou R, Wei X, Zhang X. Genome-wide analysis of WRKY gene family in the sesame genome and identification of the WRKY genes involved in responses to abiotic stresses. BMC Plant Biol. 2017;17(1):152.

35. Ning P, Liu C, Kang J, LV J. Genome-wide analysis of WRKY transcription factors in wheat (Triticum aestivum L.) and differential expression under water deficit condition. PeerJ. 2017;5:e3232. 
36. Wei Y, Shi H, Xia Z, Tie W, Ding Z, Yan Y, Wang W, Hu W, Li K. Genomewide identification and expression analysis of the WRKY gene family in cassava. Front Plant Sci. 2016;7:25.

37. Chen C, Xia R, Chen H, He Y. TBtools, a Toolkit for Biologists integrating various HTS-data handling tools with a user-friendly interface. bioRxiv. 2018:289660. https://doi.org/10.1101/289660.

38. Yue H, Wang M, Liu S, Du X, Song W, Nie X. Transcriptome-wide identification and expression profiles of the WRKY transcription factor family in Broomcorn millet (Panicum miliaceum L.). BMC Genomics. 2016;17(1):343.

39. Zhang $Y$, Wang $L$. The WRKY transcription factor superfamily: its origin in eukaryotes and expansion in plants. BMC Evol Biol. 2005;5(1):1.

40. Chen X, Li C, Wang H, Guo Z. WRKY transcription factors: evolution, binding, and action. Phytopathol Res. 2019;1(1):13.

41. Rinerson Cl, Rabara RC, Tripathi P, Shen QJ, Rushton PJ. The evolution of WRKY transcription factors. BMC Plant Biol. 2015;15:66.

42. Cannon SB, Mitra A, Baumgarten A, Young ND, May G. The roles of segmental and tandem gene duplication in the evolution of large gene families in Arabidopsis thaliana. BMC Plant Biol. 2004;4(1):10.

43. Xie T, Chen C, Li C, Liu J, Liu C, He Y. Genome-wide investigation of WRKY gene family in pineapple: evolution and expression profiles during development and stress. BMC Genomics. 2018;19(1):490.

44. Song $H$, Sun $W$, Yang G, Sun J. WRKY transcription factors in legumes. BMC Plant Biol. 2018;18(1):243

45. Bai Y, Sunarti S, Kissoudis C, Visser RGF, van der Linden CG. The Role of Tomato WRKY Genes in Plant Responses to Combined Abiotic and Biotic Stresses. Front Plant Sci. 2018;9:801.

46. Baillo EH, Kimotho RN, Zhang Z, Xu P. Transcription factors associated with abiotic and biotic stress tolerance and their potential for crops improvement. Genes (Basel). 2019;10(10):771

47. Jiang J, Ma S, Ye N, Jiang M, Cao J, Zhang J. WRKY transcription factors in plant responses to stresses. J Integr Plant Biol. 2017;59(2):86-101.

48. Banerjee A, Roychoudhury A. WRKY proteins: signaling and regulation of expression during abiotic stress responses. Sci World J. 2015;2015. https:// doi.org/10.1155/2015/807560

49. Wu X, Shiroto $Y$, Kishitani S, Ito Y, Toriyama K. Enhanced heat and drought tolerance in transgenic rice seedlings overexpressing OsWRKY11 under the control of HSP101 promoter. Plant Cell Rep. 2009;28(1):21-30.

50. Varshney RK, Shi C, Thudi M, Mariac C, Wallace J, Qi P, Zhang H, Zhao Y, Wang $X$, Rathore A. Pearl millet genome sequence provides a resource to improve agronomic traits in arid environments. Nat Biotechnol. 2017;35(10):969.

51. Goodstein DM, Shu S, Howson R, Neupane R, Hayes RD, Fazo J, Mitros T, Dirks W, Hellsten U, Putnam N, et al. Phytozome: a comparative platform for green plant genomics. Nucleic Acids Res. 2012;40(Database issue):D1178-86.

52. Lamesch P, Berardini TZ, Li D, Swarbreck D, Wilks C, Sasidharan R, Muller R, Dreher K, Alexander DL, Garcia-Hernandez M, et al. The Arabidopsis information resource (TAIR): improved gene annotation and new tools. Nucleic Acids Res. 2012;40(Database issue):D1202-10.

53. Kurata N, Yamazaki Y. Oryzabase. An integrated biological and genome information database for rice. Plant Physiol. 2006;140(1):12-7.

54. Finn RD, Clements J, Eddy SR. HMMER web server: interactive sequence similarity searching. Nucleic Acids Res. 2011;39(Web Server issue):W29-37.

55. Sievers F, Higgins DG. Clustal omega for making accurate alignments of many protein sequences. Protein Sci. 2018;27(1):135-45.

56. Sonnhammer ELL, Eddy SR, Birney E, Bateman A, Durbin R. Pfam: multiple sequence alignments and HMM-profiles of protein domains. Nucleic Acids Res. 1998;26(1):320-2.

57. Edgar RC. MUSCLE: multiple sequence alignment with high accuracy and high throughput. Nucleic Acids Res. 2004;32(5):1792-7.

58. Kumar S, Stecher G, Tamura K. MEGA7: molecular evolutionary genetics analysis version 7.0 for bigger datasets. Mol Biol Evol. 2016;33(7):1870-4.

59. Gasteiger E, Hoogland C, Gattiker A, Se D, Wilkins MR, Appel RD, Bairoch A Protein Identification and Analysis Tools on the ExPASy Server. In: Walker JM, editor. The Proteomics Protocols Handbook. Totowa: Humana Press; 2005. p. 571-607.

60. Hu B, Jin J, Guo AY, Zhang H, Luo J, Gao G. GSDS 2.0: an upgraded gene feature visualization server. Bioinformatics. 2015;31(8):1296-7.

61. Bailey TL, Boden M, Buske FA, Frith M, Grant CE, Clementi L, Ren J, Li WW, Noble WS. MEME SUITE: tools for motif discovery and searching. Nucleic Acids Res. 2009;37(Web Server issue):W202-8.
62. Wang Y, Tang H, Debarry JD, Tan X, Li J, Wang X, Lee TH, Jin H, Marler B, Guo H, et al. MCScanX: a toolkit for detection and evolutionary analysis of gene synteny and collinearity. Nucleic Acids Res. 2012;40(7):e49.

63. Team RC: R: a language and environment for statistical computing. 2013.

64. Suyama M, Torrents D, Bork P. PAL2NAL: robust conversion of protein sequence alignments into the corresponding codon alignments. Nucleic Acids Res. 2006;34(Web Server issue):W609-12.

65. Conesa A, Gotz S, Garcia-Gomez JM, Terol J, Talon M, Robles M. Blast2GO: a universal tool for annotation, visualization and analysis in functional genomics research. Bioinformatics. 2005;21(18):3674-6.

66. Lescot $M$, Déhais $P$, Thijs $G$, Marchal $K$, Moreau $Y$, Van de Peer $Y$, Rouzé $P$, Rombauts S. PlantCARE, a database of plant cis-acting regulatory elements and a portal to tools for in silico analysis of promoter sequences. Nucleic Acids Res. 2002;30(1):325-7.

67. Krishnamurthy L, Serraj R, Rai KN, Hash CT, Dakheel AJ. Identification of pearl millet [Pennisetum glaucum (L.) R. Br.] lines tolerant to soil salinity. Euphytica. 2007;158(1):179-88.

68. Jaiswal S, Antala TJ, Mandavia MK, Chopra M, Jasrotia RS, Tomar RS, Kheni J, Angadi UB, lquebal MA, Golakia BA, et al. Transcriptomic signature of drought response in pearl millet (Pennisetum glaucum (L.)) and development of web-genomic resources. Sci Rep. 2018;8(1):3382.

69. Untergasser A, Cutcutache I, Koressaar T, Ye J, Faircloth BC, Remm M, Rozen SG. Primer3-new capabilities and interfaces. Nucleic Acids Res. 2012;40(15):e115.

70. Reddy PS, Reddy DS, Sharma KK, Bhatnagar-Mathur P, Vadez V. Cloning and validation of reference genes for normalization of gene expression studies in pearl millet [Pennisetum glaucum (L.) R. Br.] by quantitative real-time PCR. Plant Gene. 2015;1:35-42.

71. Shivhare R, Lata C. Selection of suitable reference genes for assessing gene expression in pearl millet under different abiotic stresses and their combinations. Sci Rep. 2016;6:23036.

\section{Publisher's Note}

Springer Nature remains neutral with regard to jurisdictional claims in published maps and institutional affiliations.
Ready to submit your research? Choose BMC and benefit from:

- fast, convenient online submission

- thorough peer review by experienced researchers in your field

- rapid publication on acceptance

- support for research data, including large and complex data types

- gold Open Access which fosters wider collaboration and increased citations

- maximum visibility for your research: over $100 \mathrm{M}$ website views per year

At BMC, research is always in progress.

Learn more biomedcentral.com/submissions 